\title{
Einsatz von Chatbots am digitalen Büroarbeitsplatz - Eine praxisorientierte Betrachtung von Einsatzbereichen, Wirkungen und Handlungsempfehlungen
}

\author{
Raphael Meyer von Wolff $(\mathbb{D} \cdot$ Sebastian Hobert $\cdot$ Matthias Schumann
}

Eingegangen: 11. Dezember 2019 / Angenommen: 18. Februar 2020 / Online publiziert: 27. Februar 2020 (C) Der/die Autor(en) 2020

Zusammenfassung Chatbots werden heutzutage vermehrt im Unternehmen eingesetzt. Vor allem in Supportbereichen finden sich diese auf künstlicher Intelligenz und Sprachverarbeitung basierenden Technologien als Alternative für bisherige FirstLevel Kanäle. Dabei helfen Chatbots bei der Beantwortung von Kunden- und Mitarbeiterfragen und führen bereits erste kleine Aufgaben durch. Der Einsatz dieser Technologie am Büroarbeitsplatz zur Unterstützung der einzelnen Mitarbeiter ist jedoch noch kaum untersucht. Basierend auf einer empirischen Interviewstudie mit 29 Praxisvertretern aus 27 Unternehmen verschiedener Branchen soll dieses der folgende Beitrag untersuchen.

Die Ergebnisse zeigen, dass Chatbots sowohl abteilungsintern als auch abteilungsübergreifend für Aufgaben der Informationsbereitstellung sowie Prozessausführung eingesetzt werden können. Die Unternehmen beabsichtigen dabei verschiedene Wirkungen, um schlussendlich die Qualität am Arbeitsplatz zu steigern, Produktivität und Effizienz zu erhöhen, Kosten einzusparen oder das Image zu verbessern. Zusätzlich konnten sowohl technische als auch organisatorische Maßnahmen abgeleitet werden, die für einen erfolgreichen Chatboteinsatz berücksichtigt werden sollten. Der Beitrag liefert somit einen Leitfaden für zukünftige oder aktuelle Chatbotprojekte, sowohl für die Wissenschaft als auch für die Praxis.

R. Meyer von Wolff $(\bowtie) \cdot$ S. Hobert $\cdot$ M. Schumann

Professur für Anwendungssysteme und E-Business, Georg-August-Universität Göttingen, Göttingen,

Deutschland

E-Mail: r.meyervonwolff@uni-goettingen.de

S. Hobert

E-Mail: shobert@uni-goettingen.de

M. Schumann

E-Mail: mschuma1@uni-goettingen.de 
Schlüsselwörter Chatbot · Digitaler Büroarbeitsplatz · Expertenbefragung · Einsatzgebiete $\cdot$ Wirkungen $\cdot$ Handlungsempfehlungen

\title{
Application of Chatbots at the Digital Workplace-A Practice-oriented Examination of Application Areas, Effects and Guidelines
}

\begin{abstract}
Nowadays chatbots are used more and more in companies. Especially in support areas these technologies based on artificial intelligence and speech processing can be found as an alternative for previous first-level channels. Chatbots help at answering customers' and employees' questions and carrying out first small work tasks. However, the use of this technology at the office workplace to support individual employees is still considered barely and remains unknown. Based on an empirical interview study with 29 practitioners from 27 companies from a wide range of industries, the following article will examine this.

The results show that chatbots can be used both within and across departments for information provision and process execution tasks. The companies pursue various effects in order to increase quality at the workplace, increase productivity and efficiency, save costs or improve their image. In addition, both technical and organizational measures could be derived, which should be considered for a successful application of chatbots at workplaces. Thus, this article provides a guideline for current or future chatbot projects for science and practice.
\end{abstract}

Keywords Chatbot · digital workplace · expert study · application areas · effects · guidelines

\section{Bedeutung von Chatbots für den Einsatz am digitalen Büroarbeitsplatz}

In den letzten Jahren zeigt sich vermehrt ein Trend zum Einsatz von Künstlicher Intelligenz und natürlichsprachlichen Benutzungsoberflächen in Form von Chatbots zur Unterstützung der Mitarbeiter bei ihrer täglichen Arbeit (Følstad und Brandtzæg 2017; Maedche et al. 2019). Getrieben durch die generelle Digitalisierung der Gesellschaft sowie der Arbeit im Spezifischen, sind Unternehmen damit beschäftigt, bestehende papierbasierte Arbeitsweisen aufzulösen und mehr und mehr innovative, digitale Technologien einzusetzen (Byström et al. 2017; Köffer 2015; Lestarini et al. 2015; White 2012). Hierbei findet zwar eine Zunahme an Werkzeugen für die Aufgabenbearbeitung statt, jedoch behindert genau dieses wiederum die zielgerichtete Informationsbeschaffung und die tägliche Arbeit an sich. Durch die Zunahme an Anwendungssystemen und den daraus erzeugten oder abrufbaren Informationen sehen sich Mitarbeiter einem sogenannten Informationsüberschuss bzw. Anwendungssystemüberangebot ausgesetzt. Anstelle die Arbeit zu verbessern und sie produktiver bzw. effizienter zu gestalten, brauchen Mitarbeiter vielmehr mehr Zeit um Informationen zu finden, auszutauschen oder zu bearbeiten sowie schlussendlich die anstehenden Tagesaufgaben ausführen (Carayannopoulos 2018; Lebeuf et al. 2017; Russell 2012; White 2012). 
Als Lösung für diese aufkommenden Probleme wird empfohlen, nutzerorientierte Anwendungssysteme, wie Chatbots, einzusetzen, um die tägliche Arbeit zielgerichtet zu unterstützen, Aufgaben zu automatisieren sowie relevante Informationen gefiltert bereitzustellen (Følstad und Brandtzæg 2017; Reshmi und Balakrishnan 2016; Richter et al. 2018). Auch wenn sich Chatbots mittlerweile zwar im Unternehmenseinsatz befinden, z. B. im (Kunden-)Support, fehlt es an wissenschaftlichen Erkenntnissen zu diesen bisher vor allem praxisgetriebenen Vorhaben. Insbesondere der Einsatz von Chatbots am digitalen Büroarbeitsplatz ist dabei wenig betrachtet. Wie in (Meyer von Wolff et al. 2019a) abgeleitet, fehlt es sowohl an Ergebnissen zu sinnvollen Einsatzbereichen und dabei beabsichtigten Wirkungen als auch an Handlungsempfehlungen für einen Einsatz.

Um den Einsatz von Chatbots am digitalen Büroarbeitsplatz grundlegend zu untersuchen sowie bisherige Praxisergebnisse in die Wissenschaft zurückzuführen, wurde eine Befragung mit 29 Experten aus Unternehmen verschiedener Branchen durchgeführt. Anhand der Ergebnisse aus 27 geführten Interviews werden im folgenden Beitrag sowohl Einsatzmöglichkeiten inklusive dabei benötigter Aufgaben dargestellt als auch die mit dem Einsatz beabsichtigte Wirkungen aufgeführt. Außerdem werden Handlungsempfehlungen für einen erfolgreichen Einsatz aufgezeigt. Mit Hilfe der Ergebnisse wird ein Leitfaden bereitgestellt, um Chatbotprojekte erfolgreich durchzuführen und so Tätigkeiten am digitalen Büroarbeitsplatz zu unterstützen.

\section{Chatbot-basierte Unterstützung am digitalen Büroarbeitsplatz}

Erste Chatbots - auch als (Sprach-)Dialogsystem oder Conversational Agent bezeichnet - existieren bereits seit den 1960er Jahren. Die grundlegende Charakteristik hat sich dabei, unabhängig von technologischen Entwicklungen im Bereich der Künstlichen Intelligenz bzw. der Sprachverarbeitung, nicht geändert: Das Bereitstellen einer Benutzungsschnittstelle über die Anwender - sowohl mittels gesprochener als auch geschriebener Sprache - in natürlicher Art und Weise Informationen abrufen oder Aufgaben bzw. Prozesse durchführen. Hierzu stellt ein Chatbot Zugänge zu den im Unternehmen vorhandenen Datenbanken oder Anwendungssystemen sowie zu Webservices bereit. Die Funktionalitäten des Chatbots stehen dabei sowohl reaktiv als auch proaktiv sowie in Teilen autonom in Abhängigkeit von Kontext- oder Umgebungsvariablen zur Verfügung. Auch werden mitunter adaptive und selbstlernende Ansätze verfolgt, so dass Nutzerpräferenzen oder gesammelte Dialogerfahrungen in die Unterhaltungen mit einfließen (Meyer von Wolff et al. 2019b).

Getrieben durch aktuelle Entwicklungen, werden Chatbots zunehmend am digitalen Büroarbeitsplatz eingesetzt. Hierbei handelt es sich vornehmlich um die immaterielle, digitale Arbeit mit Informationen im Unternehmen, heutzutage zunehmend auch als Wissensarbeit betitelt (North 2014). Im Gegensatz zur maschinellen oder physischen Arbeit, fokussiert sich diese auf verschiedene Informationsverarbeitungsprozesse mit Hilfe von Anwendungssystemen bzw. wird diese durch Anwendungssysteme unterstützt. Zur Aufgabenausführung werden am digitalen Büroarbeitsplatz (IT-)Technologien, Prozesse und Personen sowohl innerhalb des Unternehmens als auch zwischen Unternehmen kombiniert. Der Fokus liegt dabei auf der Arbeit mit 
Informationen, einer ausgeprägten Relevanz von Kommunikation und Kollaboration zwischen beteiligten Personen sowie zwischen den Personen und den Anwendungssystemen. Unter Berücksichtigung aktueller Trends ist der Büroarbeitsplatz meistens nicht auf einen Ort beschränkt (Meyer von Wolff et al. 2019b).

\section{3 Überblick über das Studiendesign}

Um den Einsatz von Chatbots am digitalen Büroarbeitsplatz zu untersuchen und dabei sowohl Einsatzbereiche und beabsichtigte Wirkungen zu ermitteln sowie Handlungsempfehlungen abzuleiten, wurde eine explorative Interviewstudie mit Experten durchgeführt. Um einen repräsentativen Querschnitt zu ermöglichen und generalisierbare Ergebnisse abzuleiten, wurde die Branche der Befragten bewusst nicht begrenzt (Döring und Bortz 2016; Bortz und Döring 2006). Hierzu wurden branchenübergreifend 68 Experten kontaktiert, von denen 29 Experten (ca. 43\%) an den Interviews teilgenommen haben (siehe Tab. 1). Dabei wurde sichergestellt, dass sich die Experten mit der Gestaltung des digitalen Büroarbeitsplatzes unter Berücksichtigung von Chatbots beschäftigen. Zudem sollte das Unternehmen entweder bereits Chatbots einsetzen bzw. planen oder diese selber entwickeln. Als Grundlage der Interviews diente ein semistrukturierter Interviewleitfaden (Döring und Bortz 2016; Gläser und Laudel 2009; Liebold und Trinczek 2009) anhand der vier Kernkonzepte: Einsatzbereiche und Aufgaben, Wirkungen und Handlungsempfehlungen. Die Interviewauswertung erfolgte anhand der qualitativen Inhaltsanalyse nach (Mayring 2015), indem getätigte Aussagen der Experten den drei Kernkonzepten zugeordnet und anschließend weiter kategorisiert wurden.

\section{Einsatzbereiche}

Ziel des Einsatzes von Chatbots ist die Unterstützung der Mitarbeiter bei der Durchführung der täglichen Arbeit. Hierzu gilt es zu klären, welche Einsatzgebiete adressierbar sind und welche Aufgaben dabei mit einem Chatbot durchgeführt werden können. Die Ergebnisse der Studie ergaben dabei sowohl abteilungsbezogene als auch -übergreifende Einsatzgebiete (Meyer von Wolff et al. 2019b). Eine Übersicht über alle, im folgenden beschriebenen Einsatzgebiete inklusive ausgewählter Zitate und der Zuordnung identifizierter Aufgaben, kann Tab. 2 entnommen werden.

\subsection{Abteilungsbezogener Einsatz}

Aus Sicht der Abteilungen eines Unternehmens liegt überwiegend der (Kunden-)Support im Fokus der Projekte der befragten Unternehmen $(n=22)$. Anstelle des bisherigen Kontaktes über First-Level Mitarbeiter, sollen Chatbots diese erste Kontaktstelle übernehmen, um automatisiert und zu jeder Uhrzeit standardisierte Fragestellungen zu beantworten, wie z.B. bei Problemen mit Bestellungen, Urlaubsregelungen, technischen Störungen. Anstatt dass sich Support-Mitarbeiter mit simplen Fragestellungen beschäftigten, können sie sich, durch die resultierende Entlastung, auf die 
Tab. 1 Stichprobenübersicht

\begin{tabular}{|c|c|c|c|}
\hline Interview & Branche & Position & Einsatz \\
\hline 1 & Informations- \& Kommunikationstechnik & Projektleiter & O \\
\hline 2 & Informations- \& Kommunikationstechnik & Geschäftsführer & - \\
\hline 3 & Fahrzeugbau & Productowner & ○ \\
\hline 4 & Fahrzeugbau & Fachreferent & 0 \\
\hline 5 & Informations- \& Kommunikationstechnik & Online-Redakteur/Consultant & 0 \\
\hline 6 & Sonstige Dienstleistungen & Senior Manager & O \\
\hline 7 & Finanzen \& Versicherungen & Abteilungsdirektor & O \\
\hline 8 & Informations- \& Kommunikationstechnik & Teamleiter/Berater & ○ \\
\hline 9 & Finanzen \& Versicherungen & Consultant & ○ \\
\hline 10 & Finanzen \& Versicherungen & Digital Engineer & ○ \\
\hline 11 & Informations- \& Kommunikationstechnik & Geschäftsführer & $\bigcirc$ \\
\hline 12 & Informations- \& Kommunikationstechnik & Leitender KI-Architekt & ○ \\
\hline \multirow[t]{2}{*}{13} & Chemie, Pharma \& Rohstoffe & IT Service Manager & O \\
\hline & Chemie, Pharma \& Rohstoffe & IT Service Manager & \\
\hline 14 & Chemie, Pharma \& Rohstoffe & Lead IT Architect & $\times$ \\
\hline 15 & Informations- \& Kommunikationstechnik & Geschäftsführer & ○ \\
\hline 16 & Sonstiges verarbeitendes Gewerbe & Leiter Controlling und IT & $\bigcirc$ \\
\hline 17 & Sonstige Dienstleistungen & Major Account Exectuive CE & ○ \\
\hline 18 & Finanzen \& Versicherungen & $\begin{array}{l}\text { Direktor Enterprise Information } \\
\text { Management }\end{array}$ & ○ \\
\hline 19 & Sonstige Dienstleistungen & Bereichsleitung & $\bullet$ \\
\hline 20 & Informations- \& Kommunikationstechnik & Business Development Manager & ○ \\
\hline 21 & Informations- \& Kommunikationstechnik & Vorstand & $\bullet$ \\
\hline 22 & Informations- \& Kommunikationstechnik & $\mathrm{CEO}$ & ○ \\
\hline 23 & Informations- \& Kommunikationstechnik & Expert Sales Manager & 0 \\
\hline \multirow[t]{2}{*}{24} & Informations- \& Kommunikationstechnik & Accountmanager & ○ \\
\hline & Informations- \& Kommunikationstechnik & Senior Consultant & \\
\hline 25 & Sonstige Dienstleistungen & Technology Manager & ○ \\
\hline 26 & Informations- \& Kommunikationstechnik & $\begin{array}{l}\text { Bereichsleiter Workplace Ser- } \\
\text { vices }\end{array}$ & - \\
\hline 27 & Finanzen \& Versicherungen & $\begin{array}{l}\text { Projektmanager/ } \\
\text { Serviceentwickler }\end{array}$ & $\bullet$ \\
\hline
\end{tabular}

Chatbots im Einsatz

Chatboteinsatz geplant

$\times$ Chatboteinsatz nicht geplant

- keine Informationen

wichtigen bzw. komplexen Fragestellungen fokussieren. Durch Chatbots lassen sich so rund $70 \%$ der anfallenden Fragen im Servicebereich automatisch beantworten.

Im Personalwesen bietet sich der Einsatz unter anderem für die Ausschreibung von Stellen an $(n=4)$. Interessenten befragen hier den Chatbot nach offenen Stellen, Vorzügen der Anstellung oder Anforderungen an die Position sowie nach verantwortlichen Mitarbeitern. Sollten dabei einzelne Ausschreibungen in Frage kommen, kann anschließend direkt der Bewerbungsprozess über den Dialog gestartet werden. Hierbei werden benötigte Daten vom Chatbot abgefragt oder notwendige Dokumen- 


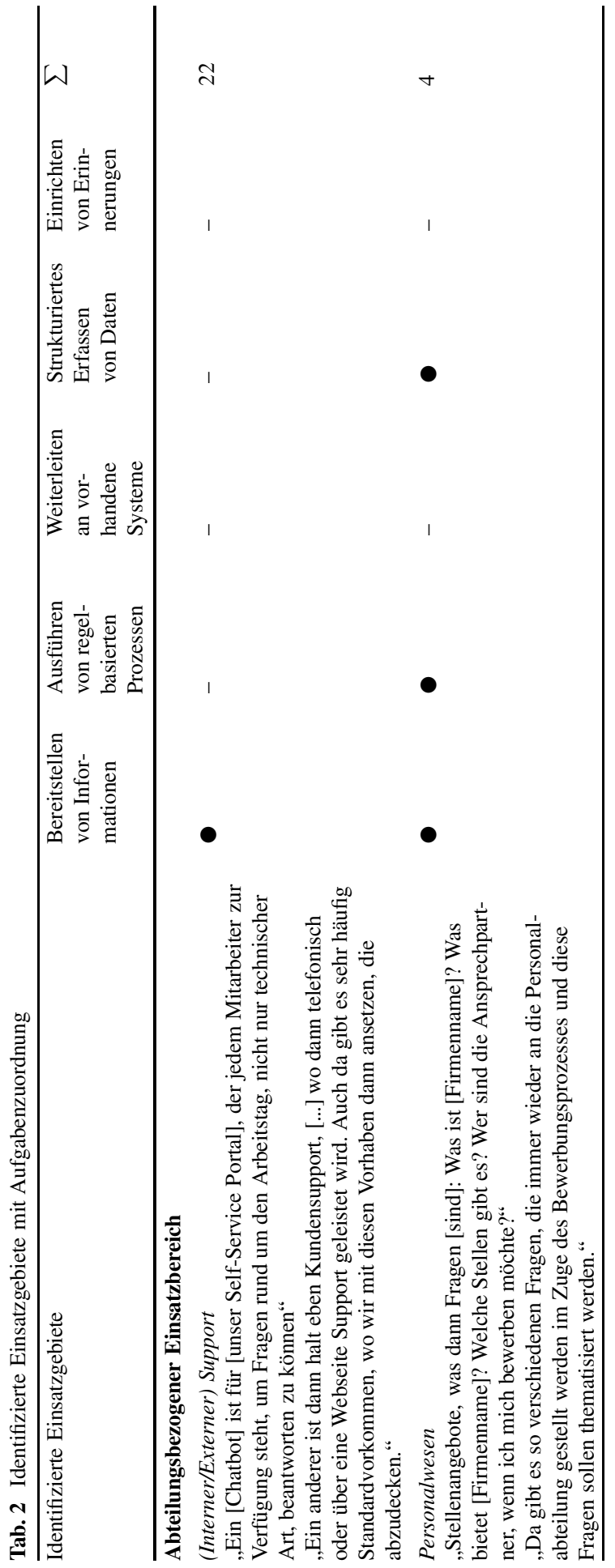




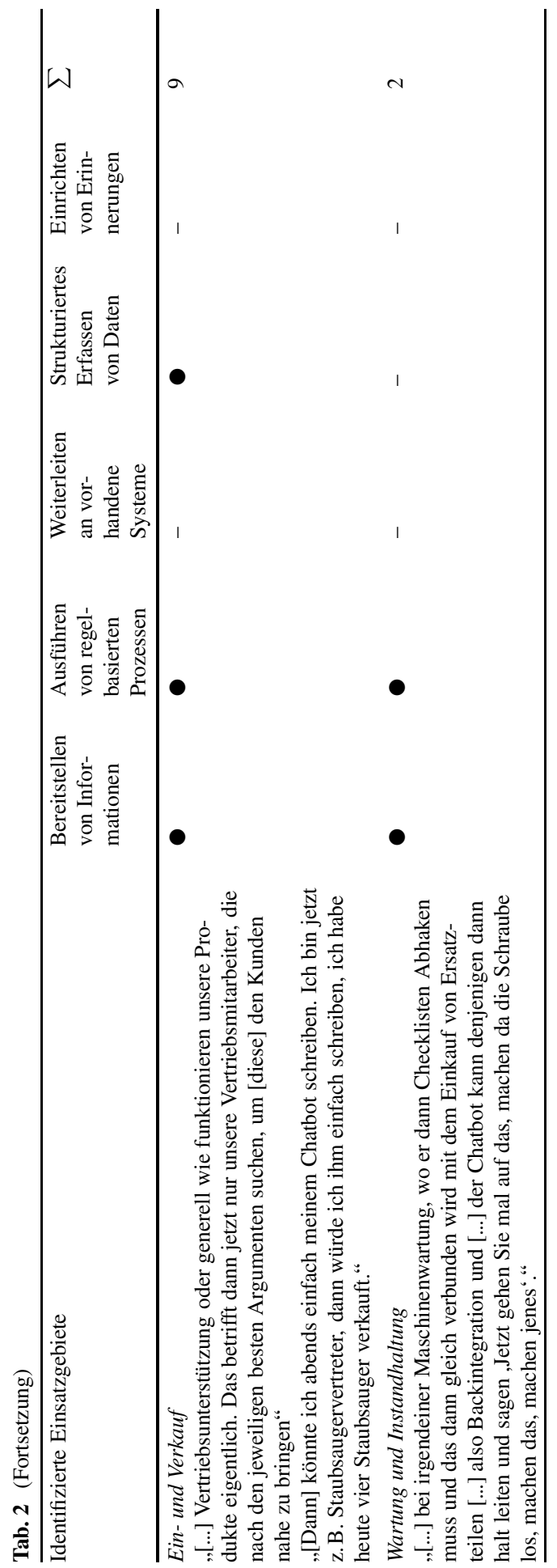




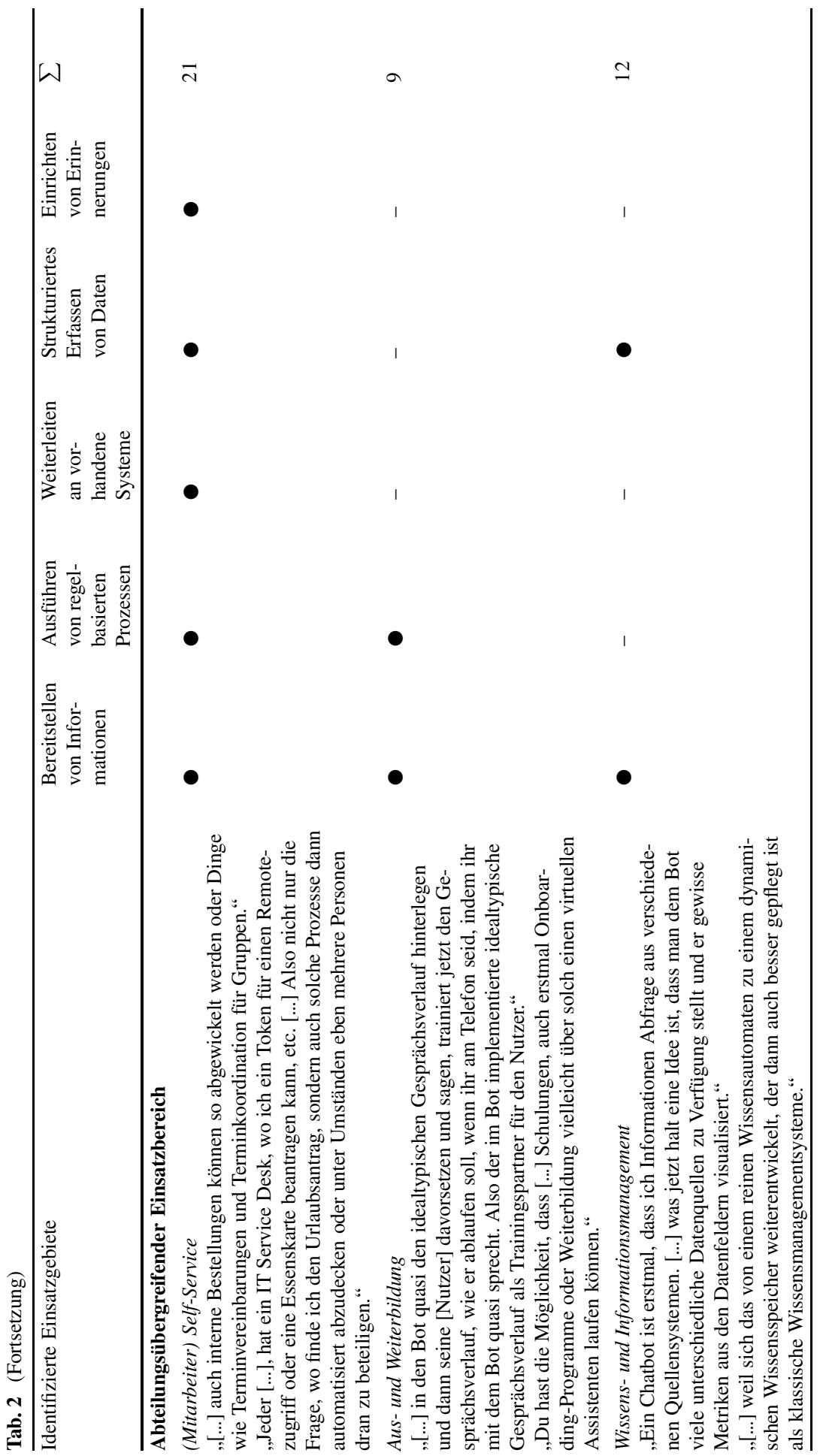


te eingeholt und der Interessent wird im direkten Gespräch durch die Bewerbung geführt. Ein Bewerber muss sich so nicht anonym durch Formulare arbeiten, sondern er erhält das Gefühl, dass er sich direkt im Gespräch bei einem Unternehmen bewirbt. Dies gestaltet den Prozess wiederum deutlich persönlicher. Resultierende Bewerbungen können sich die Mitarbeiter anschließend durch die Chatbots ausgeben lassen, welche dabei z. B. auch automatisiert vorgefiltert werden können.

Für den Ein- und Verkauf unterstützen die Systeme unter anderem den Außendienstmitarbeiter, um Verkäufe mobil zu erfassen, Erinnerungen für nachfolgende Tätigkeiten einzurichten und die Informationen an alle relevanten Anwendungssysteme weiterzugeben $(n=9)$. Auch im Unternehmen können die Verkaufsprozesse unterstützt werden. So können die Mitarbeiter den Status von Angeboten abfragen oder Angebote über natürlichsprachliche Befehle in Aufträge überführen. Außerdem können Informationen zu Produkten oder Services beschafft werden, um während einer Kundenberatung auf Kaufargumente zugreifen zu können.

Zudem eignet sich der Einsatz für Tätigkeiten der Wartung und Instandhaltung $(n=2)$. Hierbei handelt es sich zwar eher um physische Arbeit anstatt Wissensarbeit, jedoch liegen dabei Parallelen vor, z. B. die Wartung von Büroausstattungen. Chatbots können die für die Wartung benötigten Informationen bereitstellen oder durch den Prozess leiten und sicherstellen, dass keine Schritte vergessen werden. Vor allem die sprach- bzw. audiobasierte Informationsbereitstellung eignet sich dabei in Szenarien, die händische Arbeit erfordern, als Unterstützung.

\subsection{Abteilungsübergreifender Einsatz}

Bezogen auf die generelle, abteilungsunabhängige Unterstützung von Mitarbeitern wurde hauptsächlich der Self-Service Bereich von Unternehmen angesprochen $(n=21)$. Dieser weist Parallelen zum Support auf, erweitert die Funktionalitäten aber um Aufgaben der täglichen Organisation oder um unternehmens- bzw. mitarbeiterbezogene Prozesse. Anstatt nur Hilfestellungen oder Informationen bereitzustellen, sollen Chatbots vielmehr zugrundeliegende Prozesse im Dialog ausführen, z. B. das Ändern von Passwörtern oder den Antrag für eine Dienstreise bzw. deren Abrechnung. Die einfachste Form, das Hinleiten zu den benötigten Systemen, z. B. über Links oder durch das Öffnen der Anwendung, wäre hierbei bereits eine denkbare Form der Arbeitserleichterung. Sollten in den jeweiligen Prozessen Freigaben oder Abstimmungen benötigt werden, muss ein Chatbot entweder die Zustimmungen einholen oder zwischen allen Beteiligten verhandeln, z. B. im Falle einer Terminvereinbarung in einem Projektteam.

Zusätzlich wurde angesprochen, Chatbots für den Bereich der Aus- und Weiterbildung zu betreiben $(n=9)$. So können unter anderem beispielhafte Gesprächsverläufe aus dem Servicebereich im Chatbot hinterlegt werden. Durch die anschließende Interaktion mit dem System können Mitarbeiter - in der Kundenrolle - die korrekte Umgangsweise mit Kunden bzw. die optimale Antwortstrategie erlernen. Auch eignet sich die Unterstützung für das Onboarding. Neueingestellte Mitarbeiter können, ähnlich zum Supportansatz, Fragen zum Unternehmen bzw. zu spezifischem Vorgehen stellen und diese erlernen. Dabei ist es auch möglich, die beigebrachten Inhalte im Nachhinein in Form von Assessments zusätzlich abzufragen. Aber auch 
allgemeine Lerninhalte können, sofern die Form an die kleinteiligen Dialoge angepasst ist, mithilfe von Chatbots vermittelt und anschließend überprüft werden. Die Chatbottechnologie kann dabei zusätzlich adaptive Lernmaterialien für die Nutzer bereitstellen und den individuellen Lernprozess so optimal abbilden bzw. adressieren.

Auch konnte der Bereich des Wissens- und Informationsmanagements als sinnvoller Einsatzbereich identifiziert werden $(n=12)$. Wie bereits im Rahmen des Supports und des Self-Services dargestellt, stellen Chatbots eine neue Form des Zugriffs auf Unternehmensressourcen dar. Neben dem Liefern von FAQ-ähnlichen Inhalten, kann dabei auch auf dynamische Informationen zugegriffen werden, die erst zum Abfragezeitpunkt ermittelt werden, z. B. der Status der Serverlandschaft oder Umsatzzahlen bzw. Bestellungen von Kunden. Hierbei sollen die mitunter umfangreichen Wissensund Informationsbestände so verarbeitet und verdichtet werden, dass dem Nutzer eine zielgerichtete Antwort über den Dialog ausgegeben wird. Auch ist es möglich, die mit dem Chatbot geführten Dialoge für das Wissensmanagement zu verwenden, um z. B. Diskussionen oder getroffene Projektentscheidungen zu erfassen. Das klassische Wissensmanagement wird so dynamischer gestaltet und auch aktueller sowie regelmäßiger gepflegt, da die Gespräche direkt benutzt werden.

\section{Wirkungen}

Dem Einsatz von Chatbots am Büroarbeitsplatz liegen mehrere beabsichtigte Wirkungen zu Grunde. Die Auswertung der Interviews ergab dabei 14 Wirkungen in drei aufeinander aufbauenden Stufen (siehe Abb. 1). Beispielhafte Zitate zu den Wirkungen, inklusive der Nennungshäufigkeit, sind in Tab. 3 aufgeführt.

1. Stufe Der Einsatz von Chatbots besitzt drei Ausgangswirkungen. Zum einen sollen vorhandene Datenbanken und Anwendungssysteme unter einer einheitlichen Plattform zusammengeführt werden $(n=16)$. Über den Dialog sollen die verfügbaren Anwendungssysteme und Datenbanken ohne Installation einer weiteren Software bereitgestellt werden, um Aufgaben durchzuführen oder Informationen zu beziehen. Zum anderen wird eine Aufgaben-bzw. Prozessautomatisierung angestrebt $(n=15)$, wie z.B. im Falle von automatisch beantworteten Kundenfragen im Support. Wie in den Einsatzgebieten gezeigt, liegt der Fokus dabei überwiegend auf einfachen, sich wiederholenden Tätigkeiten, die viel Zeit beanspruchen sowie auf Standardfragen im Sinne von FAQs. Auch wird verfolgt, die bestehenden Services rund um die Uhr zur Verfügung zu stellen $(n=11)$, so dass z. B. Anfragen nicht von menschlichen Ressourcen abhängig sind und diese auch parallel beantwortet werden können.

2. Stufe Innerhalb der zweiten Stufe wurden weitere sechs Wirkungen ermittelt. Hierbei steht unter anderem im Fokus, die bisherigen Arbeitsumgebungen und Anwendungssysteme zu modernisieren und zu digitalisieren $(n=14)$, so dass die Unternehmenssysteme dialogbasiert, rund um die Uhr und endgeräteunabhängig bereitgestellt werden können. Zudem sollen, z. B. durch die Automatisierung, Abläufe verbessert und standardisiert werden $(n=9)$, um so unter anderem eine gleichblei- 


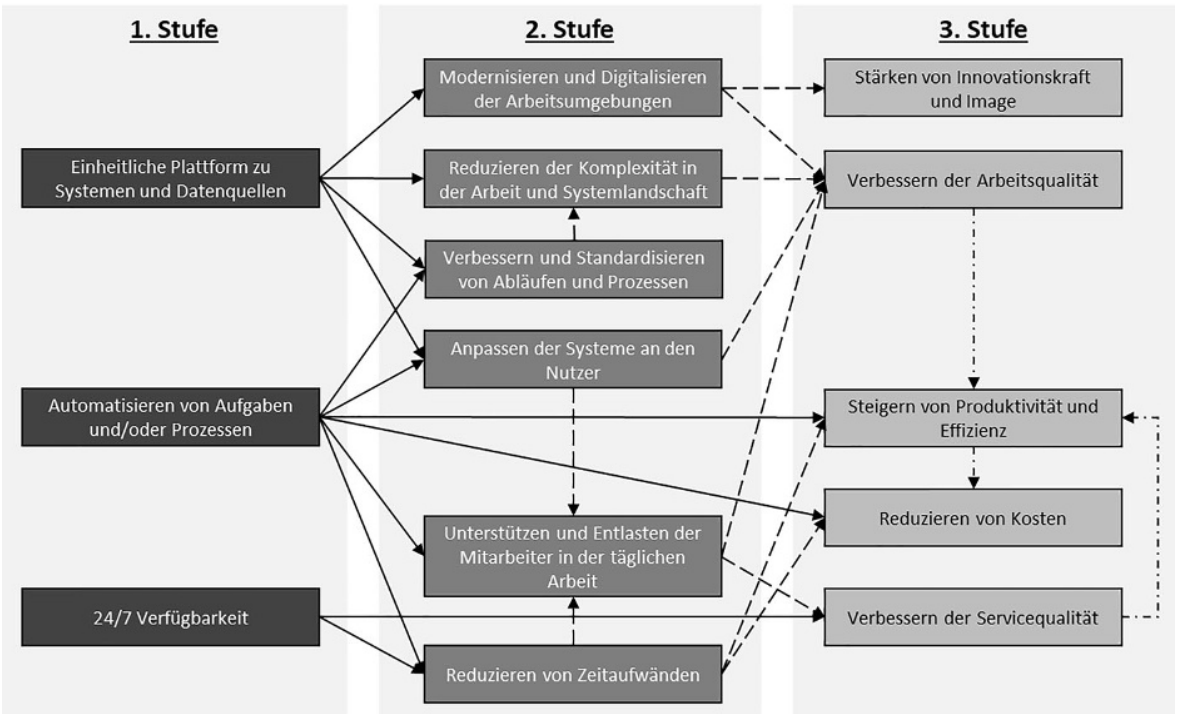

Abb. 1 Beabsichtigte Wirkungen und deren Beziehungen

bende, einheitliche Beantwortung von Fragen zu gewährleisten. Auch soll die Komplexität in der täglichen Arbeit reduziert werden $(n=3)$. Durch das Bereitstellen eines „Single Point of Contact“ zu den Unternehmenssystemen, können Mitarbeiter das gleiche System nutzen, welches auch für die Kommunikation unter der Belegschaft genutzt wird. Mitarbeiter könnten sich so sowohl untereinander austauschen als auch Unternehmenssysteme steuern. Auch wird durch den Chatbot das lang verfolgte Ziel des Anpassens der Systeme an den Nutzer adressiert $(n=12)$, indem die Mitarbeiter mittels natürlicher Sprache Funktionen ausführen können und sich nicht an die vorgegebenen Benutzungsoberflächen anpassen müssen. Mitarbeiter sollen so entlastet und optimal bei ihrer Arbeit unterstützt $(n=22)$ werden. Dabei können sich die Mitarbeiter auf wichtige Fragestellungen fokussieren, da Standardanfragen bereits automatisiert durch den Chatbot beantwortet werden. Die Arbeit wird vereinfacht und Ressourcen werden freigesetzt, die für andere Aufgaben verwendet werden können. Außerdem sollen Aufgaben möglichst umfangreich vorbereitet bzw. unterstützt werden, z. B. durch eine Teilautomatisierung. Auch wird ein Reduzieren von Zeitaufwänden mit dem Einsatz verfolgt $(n=20)$, da Nutzer von überall und unmittelbar Antworten erhalten oder Funktionen ausführen können. Zudem sind die Antworten nicht von vorhandenen Ressourcen, wie Supportpersonal abhängig.

3. Stufe Zusätzlich wurden fünf finale Wirkungen ermittelt, die ein Chatboteinsatz am Ende forciert. Dabei liegt unter anderem für das jeweilige Unternehmen das Stärken des Images im Focus $(n=4)$. Durch das Bereitstellen einer modernen innovativen Lösung, die zudem aus dem Privaten bekannt ist, sollen die Außenwirkung und die Kundenbindung sowie das Image durch neue Möglichkeiten am persönlichen Arbeitsplatz verbessert werden. Auch ergibt sich eine Verbesserung der Arbeitsqualität $(n=12)$, indem Mitarbeiter optimal unterstützt oder ihnen Aufgaben 
Tab. 3 Identifizierte Wirkungen eines Chatboteinsatzes

\begin{tabular}{ll}
\hline Identifizierte Wirkungen & $\sum$ \\
\hline
\end{tabular}

\section{Stufe}

Einheitliche Plattform zu Systemen und Datenquellen

„Aber ich glaube, dass die Kanalunabhängigkeit noch einen größeren Einfluss hat, dass man eben nicht mehr zehn oder zwanzig verschiedene Apps hat, sondern dass man [...] einfach sagen könnte ,Chatbot, ich muss [...] ein Ticket öffnen. ' und dann weiß der Chatbot, welche Daten in das Ticketsystem reingehören und kann diese eben abfragen bzw. aus der Aussage [...] extrahieren."

Automatisieren von Aufgaben und/oder Prozessen

,[...] Aufgaben, die repetitiv sind, die sich wiederholen, [...] die viel Zeit benötigen normalerweise und die aber durch so einen Bot schneller gelöst werden können, da sind Chatbots sehr gut dabei."

24/7 Verfügbarkeit

„Es soll eine Erreichbarkeit 24h 7 Tage die Woche gewährleistet werden für den Kunden.“

\section{Stufe}

Modernisieren und Digitalisieren der Arbeitsumgebungen

,[...] Adaption auf die neuen Kommunikationsgewohnheiten der viel genannten Digital Natives [...] Alle Mitarbeiter sagen, [...] dass sie lieber ein interaktives Format, wie einen Chatbot über verschiedene Messenger nutzen würden, als tatsächlich einen Telefonkontakt, sozusagen einfach die Kanalpräferenz."

Reduzieren der Komplexität in der Arbeit und Systemlandschaft

„Aber, es gibt eben diese ganzen komplexen Systeme [...], dass man über solche Interfaces, die den Anwender in gewohnter Weise kommunizieren lässt und die es ein stückweit egal werden lassen, ob es sich um einen Menschen oder um eine Maschine handelt. Solche [Chatbots] ermöglichen [es] mit dieser Komplexität [...] klar kommen.“

Verbessern und Standardisieren von Abläufen und Prozessen

„[...] Transparenz in den Prozessen und eine gewisse Standardisierung, weil wenn einmal auf eine bestimmte Art und Weise ein Fall gelöst wurde und der nächste User ruft an und [...] hat das gleiche Problem dann kann man sicher sein, dass wieder der gleiche Lösungsweg beschritten wird.“

Anpassen der Systeme an den Nutzer

„Es wird zwar immer wieder versprochen, dass die Technologie sich uns anpasst, aber das ist ja noch immer nicht der Fall und das versprechen Chatbots zu tun, weil sie sich dem Nutzer anpassen und ihm die Information und die Anwendungsfälle zur Verfügung stellen, die er braucht $[\ldots]^{*}$

Unterstützen und Entlasten der Mitarbeiter in der täglichen Arbeit

„,...] dieses Thema Conversational UI, wo der [Chatbot] die Führung des Nutzers übernehmen kann und ihn gezielt nach Informationen fragen und durch einen Baum von Lösungsmöglichkeiten navigieren kann.“

„Da würde ich eine solche Technologie eher in dem Bereich sehen, dass dort entlastet wird, um Kapazitäten frei zu schaffen für Neues.“

Reduzieren von Zeitaufwänden

„Wir sehen das Thema Geschwindigkeit in der Beantwortung, wo eben manche einfachen Anliegen auch lange liegen bleiben können, wenn ein großvolumiges Anfragevolumen da ist, wird man hier schneller bedient." 
Tab. 3 (Fortsetzung)

\begin{tabular}{l}
\hline Identifizierte Wirkungen \\
\hline 3. Stufe \\
Stärken von Innovationskraft und Image \\
„Aber die auslösenden Elemente sind momentan tatsächlich auf der vertrieblichen Seite die \\
Themen Kundenbindung, moderne innovative Kommunikationskanäle, Customer Journey, \\
dort irgendwo positiv zu punkten.“ \\
Verbessern der Arbeitsqualität \\
„Auf der anderen Seite, [...] rückt der Mensch als solches immer mehr in den Fokus, das \\
heißt dort ein möglichst angenehmes Arbeitsumfeld zu schaffen und da gehört für mich, [...] \\
da gehört sowas zukünftig dazu.“ \\
Steigern von Produktivität und Effizienz \\
„[...] natürlich ein Chatbot auch ein echter Effizienzfaktor, wenn der in der Lage ist, dass \\
Vorgänge schon vorab selbstständig zu erledigen bevor da ein echter Mitarbeiter dran muss \\
und sich darum kümmert, [...]“ \\
Reduzieren von Kosten \\
„Natürlich ist der Nutzen auf der einen Seite Kosteneinsparung, egal, ob wir das im Kun- \\
denservice einsetzen, [...] oder intern [...], indem die Leute sich selber [...] bzw. durch den \\
Chatbot die Informationen beschaffen können.“ \\
Verbessern der Servicequalität \\
„[...] ein Chatbot ist permanent erreichbar unabhängig von normalen Arbeitszeiten. Damit \\
erhöht sich die Servicequalität.“
\end{tabular}

durch (Teil-)Automatisierung abgenommen werden. Vor allem durch die 24/7 Verfügbarkeit sowie die schnelle Reaktion auf Anfragen wird eine gesteigerte Servicequalität beim Chatboteinsatz erwartet $(n=2)$. Auch lässt sich so eine Steigerung von Produktivität und Effizienz in der täglichen Arbeit jeden Mitarbeiters ableiten $(n=13)$. Dies hat ebenfalls Kosteneinspareffekte zum Ziel, da Prozesse zumindest teilautomatisiert ablaufen sollen $(n=19)$. Dabei stellen Kosteneinsparungen jedoch immer nur eine Folge des gesamten Vorhabens dar und wurden nicht als primäre Wirkung von den Befragten genannt.

\section{Handlungsempfehlungen}

Neben Einsatzbereichen und beabsichtigten Wirkungen gilt es, Maßnahmen für einen erfolgreichen Einsatz von Chatbots am Arbeitsplatz zu ermitteln. Hierzu wurden die Experten zu Herausforderungen befragt, aus welchen anhand von argumentativ-deduktiven Schlussfolgerungen Handlungsempfehlungen abgeleitet wurden. Diese technischen und organisatorischen Handlungsempfehlungen werden nachfolgend thematisiert.

\subsection{Technische Handlungsempfehlungen}

Essentiell für den Betrieb von Chatbots ist es, dass diese auf eine umfangreiche und aktuelle Datenbasis zurückgreifen können. Hierzu sollte bereits im Vorfeld des Einsatzes mit dem Aufbau von NLP-fähigen Datenbeständen begonnen werden. Oftmals existieren zwar, z. B. für den Fall des Supportes, Standardantworten, jedoch fehlt das 
Beziehungswissen, um Inhalte Dialogbasiert abrufbar zu machen. Hier bietet es sich an, auch die Mitarbeiter einzubeziehen, da diese oftmals die umfangreichsten Kenntnisse des Anwendungsfalls besitzen. Entscheider sollten auch berücksichtigen, dass bestehende (operative) Datenbanken zusätzlich nicht immer für einen NLP-Einsatz ausgerichtet sind, z. B. aufgrund von fehlenden Schnittstellen oder unpassenden Datenformaten. Der Aufwand hört dabei aber nicht beim Bereitstellen auf. Vielmehr muss das Wissen des Chatbots kontinuierlich gepflegt und (nach-)trainiert werden. Nur so wird sichergestellt, dass Chatbots die Nutzeranfragen verarbeiten können und entsprechende Antworten bzw. Lösungen bereitstellen. Es bietet sich unter anderem an, z. B. durch Chatbots freigesetzte Kapazitäten hierfür einzuplanen. Fehler oder nicht verstandene Aussagen sollten zusätzlich zeitnah korrigiert werden, denn merkt ein Nutzer, dass der Chatbot auch nach mehreren Tagen noch so limitiert ist wie zu Beginn, wird er die weitere Nutzung eher einstellen als weiterführen.

Auch hinsichtlich der Funktionalität gilt es, einige Aspekte zu berücksichtigen. So sollte ein Chatbot, gemäß den global agierenden Unternehmen sowie deren Belegschaft, möglichst mehrsprachig ausgelegt sein. Dies bedeutet jedoch auch, dass die verwendeten Datenbestände entsprechend zu entwickeln sind. Dabei gilt es, nicht nur die verschiedenen Sprachen, sondern mitunter auch Dialekte zu berücksichtigen, so dass ein Chatbot sie verarbeiten kann. Zusätzlich muss der Chatbot eine gewisse Robustheit gegenüber Eingabefehlern unterstützen. So sollte auch trotz Tippfehler oder falscher Schreibweisen ein Anfragender eine Antwort bzw. Lösung erhalten. Wenn ein Chatbot jedoch auch nach mehrmaligen Nachfragen immer noch keine Antwort liefern kann, bietet sich eine Weiterleitung an einen realen Mitarbeiter an, um dem Anfragenden trotzdem zu helfen. Im schlimmsten Falle würde dieses sonst zu Unzufriedenheit oder Ablehnung führen. Bei diesem Weiterleiten sollte zusätzlich der bisherige Chatverlauf mit übergeben werden, so dass der Anfragende nicht erneut alle Inhalte angeben muss. Zudem sollten solche Fälle im Nachgang analysiert werden, um den Chatbot entsprechen zu trainieren.

Damit ein Chatbot nicht nur ein weiteres Anwendungssystem in der Systemlandschaft darstellt, sind bereits von Beginn an Integrationsaspekte zu berücksichtigen. Die Integration in die Systemlandschaft spielt dabei eine besondere Rolle. Wie bereits hinsichtlich der Datenbestände dargestellt, werden Schnittstellen benötigt, so dass ein Chatbot direkt die Datenbanken oder andere angeschlossene Systeme ansprechen kann. Im Umkehrschluss müssen Chatbots auch in die Oberflächen der verfügbaren Systeme eingebunden werden, um die Zugänglichkeit zu den bereitgestellten Funktionen zu fördern.

Eine Zusammenfassung der technischen Handlungsempfehlungen mit ausgewählten Zitaten zu genannten Herausforderungen ist in Tab. 4 abgebildet.

\subsection{Organisatorische Handlungsempfehlungen}

Um den Einsatz von Chatbots erfolgreich durchzuführen, ist es notwendig, die zukünftigen Benutzer einzubeziehen. Auch wenn sich Chatbots zwar an den Nutzer anpassen sollen, sind, insbesondere bei der älteren Belegschaft, die Art und Weise der neuen Benutzungsoberfläche nicht für jeden bekannt. Es bietet sich hier an, Schulungen innerhalb der Belegschaft bzw. unter den zukünftigen Nutzenden durch- 
Tab. 4 Technische Handlungsempfehlungen

\begin{tabular}{ll}
\hline Kategorie & Handlungsempfehlungen \\
\hline Datenbasis & Aufbau NLP-fähiger Datenbestände \\
& Abbilden von Beziehungswissen innerhalb der \\
& Daten(-banken) \\
Kontinuierliche Pflege und Training der Wissens- & basis
\end{tabular}

„Das hat Auswirkungen auf das Anlernen eines Chatbots, die fortlaufende Datenpflege, was unglaublich umfassend wird je größer dieser Chatbot.“

„Dort geht es darum Daten zur Verfügung zu stellen und Daten vor allen Dingen standardisiert zur Verfügung zu stellen und das wäre so die technologische Herausforderung [...].“

„Zu den ersten Herausforderungen gehört das Thema Trainingsaufwand. Also es ist so, dass eine Chatbotkomponente wenn man sie frisch in Betrieb nimmt, selbst mit vernünftiger Aufbereitung von zum Beispiel FAQ Wissen, immer noch zahlreiche Wendungen unserer Kommunikation nicht versteht. Das heißt, es gibt einen großen vorab Aufwand und einen großen während dessen Aufwand.“

Funktionalität

Unterstützen von Mehrsprachigkeit in der Interak-

tion

Weiterleiten an reale Mitarbeiter bei Wissenslücken

„[...] die Schwierigkeit darzustellen, wie schwer es ist, ein wirkliches dynamisches, natürliches Gespräch hinzukriegen. Und das gilt nicht nur für normale Gespräche, sondern auch für alle Prozesse, die man abbilden kann. Also du kannst nur einen bestimmten Bereich von Dynamik wirklich in einem Chatbot abbilden.“

„[...] Komplexität könnte um die Sprache herum existieren. Wenn Sie ein multinationales Unternehmen sind, sind Ihre Chatbots viel, viel komplexer, weil Sie Dinge in vielen verschiedenen Sprachen vorhalten müssen.“

Systemlandschaft

Daten- und Prozessintegration mit der System-
landschaft
Oberflächenintegration in die vorhandenen Syste-
me

„Wir benutzten bestehende Schnittstellen, aber immer müssen die Schnittstellen auch neu gemacht werden und die müssen eben auch gepflegt werden. Also, das ist dann schon auch, wenn man tief in die Unternehmenssysteme reingeht, dann ist das auch schon ganze Menge Anpassungsaufwand innerhalb der Unternehmens IT.“

„Du hattest angesprochen über bestehende Chatprogramme vielleicht was zu machen, [...] dass wir hier mit unserem Skype oder Jabber direkt mit dem Chatbot dann auch kommunizieren können, das sehe ich noch nicht."

zuführen, so dass die neuen Funktionsweisen vermittelt werden können. Vor allem die Aufgabenausführung anhand eines Gesprächs und die dadurch zu lernende Syntax gilt es hierbei zu adressieren. Hierzu können unter anderem die Chatbotvorhaben mit einem Change- \& Erwartungsmanagement begleitet werden. So können von Beginn an zu hohe Erwartungen an die Funktionalität bzw. nicht gesehene Vorteile der Nutzenden direkt angesprochen werden, um eine Akzeptanz für die neuen Systeme innerhalb der Belegschaft aufzubauen. Die Angst um einen Arbeitsplatzverlust durch die Übernahme des First-Level-Supports durch einen Chatbot kann z. B. gemindert werden, da freigesetzte Kapazitäten für neue (wichtige) Aufgaben genutzt werden.

Auch sollten entsprechende Projekte nur anhand eines geplanten Vorgehens umgesetzt werden, welches oftmals für Chatbots noch nicht im Unternehmen etabliert ist. Zum einen müssen Anwendungsfälle gefunden und definiert werden, für die ein Chatbot praktikabel ist. Meistens erzielen Chatbots heutzutage nur bei einem ange- 
Tab. 5 Organisatorische Handlungsempfehlungen

\begin{tabular}{ll}
\hline Kategorie & Handlungsempfehlungen \\
\hline Einbeziehen der Benutzer & Benutzerschulungen für die neue Interaktionsme- \\
thode & Change- \& Erwartungsmanagement unter den \\
& zukünftigen Nutzern
\end{tabular}

„Eine weitere Limitation ist, die Hemmschwelle den Chatbot tatsächlich auch zu benutzen. Wenn ich in meine typischen Arbeitsraster verfallen bin und ich weiß ganz genau, was ich benutzen muss, wie das funktioniert, dann brauche ich normalerweise keine Chatbots. [...] Deswegen sind [...] Mitarbeiter, die schon länger im Unternehmen sind, eher skeptisch gegenüber Chatbots.“

„Ich benutze [Google Assistent] fast gar nicht, [...] weil ich immer viel zu viel nachdenken muss, wie ich eine Frage formulieren kann, damit er die versteht. Also ich kann nicht einfach [mit dem reden ...]. Solange ich das nicht kann, [...] kann ich es auch gleich wieder über das alte IT System machen oder jemanden anrufen."

\title{
Geplantes Vorgehen
}

Finden \& Abgrenzen von sinnvollen Anwendungsfällen

Definition und Reorganisation der abzubildenden Prozesse

Ressourcen zusichern \& Aufbau von verantwortlichen Abteilungen

„Die kritischste Herausforderung ist für mich tatsächlich der Use Case. [...] und der Use Case ist immer davon abhängig ob die Daten und die Systeme vorhanden sind, die ich brauche um den Use Case abzubilden. [...] es gibt einfach Use Cases, die kann ich über ein grafisches Interface deutlich schneller abbilden [...] Es wird aber Use Cases geben, wo ich gehemmt bin, durch [...] eine normale UI [...]“ „Das haben wir auch schon probiert, [...] vielleicht können wir über den schlecht designten Prozess, den wir derzeit haben, Automatisierung abbilden, sodass wir da schneller werden. Ne, funktioniert nicht“" „Das Management unterstützt uns eigentlich durch die Bereitstellung der Budgets und die Formulierung des Wunsches, dass man das haben will. Zieht sich dann aber auch schon eher zurück.“

\section{Datenschutz und -sicherheit}

\author{
Sicherstellen der DSGVO \\ Auswahl eines Anbieters vs. Entwickeln eigener \\ Chatbots
}

„Also wenn wir nach Deutschland schauen, ist das allererste, was mir einfällt, Datenschutzprobleme. Das Thema Datenschutz ist extrem wichtig bei diesem Themengebiet, zumindest um dieses Thema in Deutschland zu etablieren.“

„[...] nächste Herausforderung wer ist verantwortlich für das Wissen was wir dort drinnen habe? Geben wir das einem externen Dienstleister müssten wir dem all unser Wissen geben. Wollen wir dem unser Wissen geben über [Firmenname]? Wer ist verantwortlich, dass dieses Wissen gepflegt wird? Wer ist verantwortlich, dass das was da drin ist auch immer noch aktuell bleibt?“"

messen hohen Frageaufkommen, welches sinnvoll automatisiert werden kann, sowie in einem thematisch abgeschlossenen Bereich, einen Mehrwert. Als Devise bietet es sich an, mit einem sinnvollen kleinen Anwendungsfall anzufangen, welcher über eine kritische Nutzenmasse bzw. Fragenaufkommen verfügt, um hierrüber Akzeptanz unter den Nutzern aufzubauen. Zum anderen ist es notwendig, die Prozesse zu untersuchen und zu definieren, die in Zukunft durch einen Chatbot abgebildet werden. Oftmals müssen diese dabei zunächst einer Prozessreorganisation unterworfen werden, da sie in der aktuellen Form nicht eins zu eins mit einem Chatbot umgesetzt werden können. Neben dem Schaffen der ablauforganisatorischen Grundlagen, müssen zusätzlich im Unternehmen Ressourcen bereitgestellt bzw. Abteilungen aufgebaut werden, die für die Chatbotprojekte verantwortlich sind. So werden Mitarbeiter be- 
nötigt, die mit dem kontinuierlichen Training der Chatbots beauftragt sind, und es muss fehlendes Wissen in den Abteilungen erzeugt werden.

Weiterhin ist es notwendig, Datenschutz und -sicherheit zu gewährleisten. Durch die Freitexteingaben der Nutzer im Dialog besteht immer die Gefahr, dass personen- bzw. unternehmensbezogene Daten eingegeben werden. Hier gilt es die DSGVO sicherzustellen, bzw. entsprechende Hinweise im Dialog anzuzeigen. Kritisch ist dabei auch, wie bzw. wo die Eingaben verarbeitet werden. Je nach Anbieter werden mitunter nicht-europäische Server verwendet. Unternehmen sollten somit zwischen externem Anbieter oder Inhouse-Betrieb abwägen. Ersteres führt mitunter zu Abhängigkeiten, da Chatbots bei einem zukünftigen Anbieterwechsel nicht einfach übertragen werden können. Letzteres hingegen erfordert aber wiederum den Aufbau entsprechender Infrastrukturen und von Know-how im Unternehmen.

Eine Zusammenfassung der organisatorischen Handlungsempfehlungen mit ausgewählten Zitaten zu genannten Herausforderungen ist in Tab. 5 abgebildet.

\section{Schlussbetrachtung}

Die Ergebnisse der Expertenstudie belegen die anfangs dargestellten Potentiale von Chatbots für den Einsatz am digitalen Büroarbeitsplatz. Wie gezeigt werden konnte, existieren dabei sieben Einsatzbereiche sowohl abteilungsbezogen als auch abteilungsübergreifend. Dabei wurden zusätzlich fünf Aufgaben identifiziert, die von Chatbots übernommen werden können. Chatbots sollen dabei aber nicht die Einsatzbereiche übernehmen sondern durch ihre Funktionalität die Einsatzbereiche entsprechende im Rahmen der fünf Aufgaben unterstützen. Über alle Einsatzgebiete hinweg stellt insbesondere das Bereitstellen von Inhalten oder Dokumenten die wichtigste Funktionalität dar. Aber auch das Ausführen von Prozessen und das strukturierte Erfassen von Daten sollte als Funktion bedacht werden. Je nach Einsatzbereich bzw. tatsächlicher Aufgabe ist dabei aber zu prüfen, welche Funktionen tatsächlich bereitzustellen sind, so dass die hier aufgestellten Übersichten lediglich als Ansatzpunkte zu sehen sind. Zusätzlich wurde ermittelt, dass der Support der fokussierteste Einsatzbereich ist, da es hier nur um das Bereitstellen von Informationen geht. Eine tiefergehende Integration in die Unternehmensprozesse ist dabei nicht notwendig. Gegensätzlich hierzu ist der Self-Service, da je nach Implementierung alle ermittelten Aufgaben von Relevanz sein können.

Als Argumente für einen Einsatz wurden insgesamt 14 Wirkungen angesprochen. Die Vorhaben stützen sich vor allem auf den einheitlichen Zugang zu Systemen, (Teil-)Automatisieren von Aufgaben sowie eine rund um die Uhr Verfügbarkeit. Schlussendlich sollen Chatbots die Innovationskraft des Unternehmens steigern, die Arbeits- und Servicequalität erhöhen, die Produktivität und Effizienz der Mitarbeiter verbessern sowie Kosten reduzieren. Anhand der Beziehungen konnten mehrere mögliche Pfade identifiziert werden, wie ein Chatbot positive Wirkungen am digitalen Arbeitsplatz entfalten kann.

Auch wenn die Entwicklung eines ersten Chatbot-Prototypen schnell durchgeführt ist, gilt es mehrere Maßnahmen zu treffen, damit der Einsatz am Ende tatsächlich erfolgreich verläuft. Hierzu liefert der Beitrag mehrere Handlungsempfeh- 
lungen. Dabei wurden unter anderem sieben technische sowie neun organisatorische Handlungsempfehlungen durch die Interviewpartner angesprochen. Insbesondere müssen organisatorische Maßnahmen getroffen werden, die eine Einführung begleiten.

In der vorliegenden Studie wurden Experten aus national und global agierenden deutschen Unternehmen zu Einsatzmöglichkeiten und notwendigen Aufgaben, Wirkungen sowie Handlungsempfehlungen beim Einsatz von Chatbots am digitalen Büroarbeitsplatz befragt. Die Ergebnisse basieren dabei auf den Experten und Ihrer Auskunftsbereitschaft sowie auf den daraus basierenden Auswertungen der Autoren. Um dabei entstehende Limitationen zu adressieren, wurde ein strukturiertes Vorgehen verfolgt. Somit können die Ergebnisse die bisherig verbreitete Etablierung von natürlichsprachlichen Benutzungsschnittstellen fundieren und sowohl Entscheidern als auch Entwicklern Ansatzpunkte für potentielle eigene Chatbotvorhaben bieten.

Funding Open Access funding provided by Projekt DEAL.

Open Access Dieser Artikel wird unter der Creative Commons Namensnennung 4.0 International Lizenz veröffentlicht, welche die Nutzung, Vervielfältigung, Bearbeitung, Verbreitung und Wiedergabe in jeglichem Medium und Format erlaubt, sofern Sie den/die ursprünglichen Autor(en) und die Quelle ordnungsgemäß nennen, einen Link zur Creative Commons Lizenz beifügen und angeben, ob Änderungen vorgenommen wurden.

Die in diesem Artikel enthaltenen Bilder und sonstiges Drittmaterial unterliegen ebenfalls der genannten Creative Commons Lizenz, sofern sich aus der Abbildungslegende nichts anderes ergibt. Sofern das betreffende Material nicht unter der genannten Creative Commons Lizenz steht und die betreffende Handlung nicht nach gesetzlichen Vorschriften erlaubt ist, ist für die oben aufgeführten Weiterverwendungen des Materials die Einwilligung des jeweiligen Rechteinhabers einzuholen.

Weitere Details zur Lizenz entnehmen Sie bitte der Lizenzinformation auf http://creativecommons.org/ licenses/by/4.0/deed.de.

\section{Literatur}

Bortz J, Döring N (2006) Forschungsmethoden und Evaluation. Springer, Heidelberg

Byström K, Ruthven I, Heinström J (2017) Work and information: which workplace models still work in modern digital workplaces? Inf Res 22(1):CoLIS paper 1651. http://InformationR.net/ir/22-1/colis/ colis 1651.html

Carayannopoulos S (2018) Using chatbots to aid transition. Int Jnl Info Learn Tech 35:118-129. https:// doi.org/10.1108/IJILT-10-2017-0097

Döring N, Bortz J (2016) Forschungsmethoden und Evaluation in den Sozial- und Humanwissenschaften. Springer, Berlin, Heidelberg

Følstad A, Brandtzæg PB (2017) Chatbots and the new world of HCI. interactions 24:38-42. https://doi. org/10.1145/3085558

Gläser J, Laudel G (2009) Experteninterviews und qualitative Inhaltsanalyse als Instrumente rekonstruierender Untersuchungen. VS, Wiesbaden

Köffer S (2015) Designing the digital workplace of the future-what scholars recommend to practitioners. 36th International Conference on Information Systems, S 1-21

Lebeuf C, Storey M-A, Zagalsky A (2017) How software developers mitigate collaboration friction with Chatbots talking with conversational agents. In: Collaborative action workshop at the 20th ACM conference on computer-supported cooperative work and social computing, S 1-6

Lestarini D, Raflesia SP, Surendro K (2015) A conceptual framework of engaged digital workplace diffusion. 9th International Conference on Telecommunication Systems Services and Applications, S 1-5 
Liebold R, Trinczek R (2009) Experteninterviews. In: Kühl S (Hrsg) Handbuch Methoden der Organisationsforschung. Quantitative und qualitative Methoden. VS, GWV, Wiesbaden, S 32-56

Maedche A, Legner C, Benlian A, Berger B, Gimpel H, Hess T, Hinz O, Morana S, Söllner M (2019) AI-based digital assistants; opportunities, threats, and research perspektives. Bus Inf Syst Eng 61:535-544. https://doi.org/10.1007/s12599-019-00600-8

Mayring P (2015) Qualitative Inhaltsanalyse; Grundlagen und Techniken. Beltz, Weinheim

Meyer von Wolff R, Hobert S, Schumann M (2019a) How may I help you? - state of the art and open research questions for chatbots at the digital workplace. Proceedings of the 52nd Hawaii International Conference on System Science, S 95-104

Meyer von Wolff R, Masuch K, Hobert S, Schumann M (2019b) What do you need today? —an empirical systematization of application areas for chatbots at digital workplaces. Proceedings of 25 th Americas Conference on Information Systems, S 1-10

North K (2014) Wissensarbeit - Wertschöpfung im Wandel. In: Antoni CH, Friedrich P, Haunschild A, Josten M, Meyer R (Hrsg) Work-Learn-Life-Balance in der Wissensarbeit: Herausforderungen, Erfolgsfaktoren und Gestaltungshilfen für die betriebliche Praxis. Springer, Wiesbaden, S 21-39

Reshmi S, Balakrishnan K (2016) Implementation of an inquisitive chatbot for database supported knowledge bases. Sādhanā 41:1173-1178

Richter A, Heinrich P, Stocker A, Schwabe G (2018) Digital work design. Bus Inf Syst Eng 60:259-264. https://doi.org/10.1007/s12599-018-0534-4

Russell DM (2012) Ubiquitous search for smart workspaces. Univ Access Inf Soc 11:337-344. https://doi. org/10.1007/s10209-011-0244-X

White M (2012) Digital workplaces: Vision and reality. Bus Inf Rev 29:205-214. https://doi.org/10.1177/ 0266382112470412 